\title{
Análisis lingüístico clínico de las alteraciones fonológicas en el niño: un estudio de caso
}

\section{Clinical linguistic analysis of phonological alterations in children: A case study}

\author{
Andrés Casadiego \\ Benemérita Universidad Autónoma \\ de Puebla \\ Josaphat Guillén \\ Benemérita Universidad Autónoma \\ de Puebla \\ Yulia Solovieva \\ Benemérita Universidad Autónoma \\ de Puebla \\ Regina Machinskaya \\ Benemérita Universidad Autónoma \\ de Puebla
}

Contacto con el autor: Andrés Casadiego

Puebla - México

Correo-e: andres.casadiegom@gmail.com

Recibido: 04/04/2019

Aceptado: 01/11/2019

\begin{abstract}
RESUMEN
En los últimos años, dentro del ámbito clínico se ha pugnado por la integración de un marco teórico inter/multidisciplinar que permita explicar de forma integral las alteraciones del lenguaje. Esto adquiere mayor relevancia cuando se proponen categorías disgnósticas que se limitan a describir el daño y no a explicar las causas subyacentes. Así, los objetivos de este trabajo son: (1) identificar los factores neurofisiológicos y neuropsicológicos alterados, a partir de la perspectiva neuropsicológica de Luria, en un niño con problemas de lenguaje y (2) realizar un análisis lingüístico de los errores fonológicos para determinar la relación existente entre ambos elementos. El método que se siguió fue un estudio de caso -de tipo descriptivo- donde se realizó un análisis cualitativo de los déficits fonológicos del paciente. Los resultados muestran que el niño presentaba un amplio rango de alteraciones fonológicas: asimilación, sustitución, omisión e inserción, todos ellos relacionados con el mecanismo de análisis y síntesis cinestésico y la organización secuencial de movimientos y acciones. Estos resultados sugieren que existe una relación entre los factores neuropsicológicos alterados y el tipo de error fonológico presentado. Se propone aquí, que los déficits del lenguaje pueden explicarse en mayor medida si se considera un enfoque neuropsicológico y lingüístico. Finalmente, la inclusión de la lingüística al campo clínico fonoaudiológico permitiría explicar los fenómenos observados de manera más integral.
\end{abstract}

Palabras clave: fonología, trastorno de lenguaje, lingüística clínica, EEG, neuropsicología.

\section{ABSTRACT}

Over the last years, the need of an inter/multidisciplinary framework has been advanced within clinical contexts that might integrally explain language alterations. This need is even more relevant when diagnostic categories that have been proposed describe mostly the damage, but they do not explain underlying causes. Thus, the aims of this work are: (1) identifying the altered neurophysiological and neuropsychological factors, from a Lurian neuropsychology perspective, in a child with language deficits and, (2) carry out a linguistic analysis of phonological errors to determine the relation between both facts $A$ single-case study was implemented, and qualitative analysis was conducted to describe the child's phonological deficits. Results showed that the child had a wide range of phonological alterations: assimilation, substitution, omission, and insertion, all of them related with a deficit in kinesthetic analysis and synthesis mechanism, and sequential organization of movements and actions. These findings suggest that there is a relation between altered neuropsychological factors and type of phonological error observed. We propose that language deficits can be explained better if we consider a neuropsychologic and linguistic approaches. Finally, if a linguistic approach is including in the speech pathology clinical context, we can expect explain the observed phenomenon in a more integral way.

Keywords: phonology, language disorder, clinic linguistic, EEG, neuropsychology. 


\section{Introducción}

En los últimos años, dentro del ámbito clínico, se ha pugnado por la consolidación de un marco teórico interdisciplinario que permita abordar el estudio de las patologías del lenguaje de manera integral. En este sentido, se puede considerar que los primeros intentos para desarrollar un marco teórico de esta naturaleza se remontan a Luria y Jakobson, quienes abogaron por un análisis estructural de los déficits lingüísticos en el ámbito clínico. Actualmente, a pesar de que el valor de los estudios inter y/o multidisciplinares no está en duda, aún sigue estando pendiente la consolidación de un marco teórico neuropsicológico-lingüístico que pueda coadyuvar a la investigación clínica de las alteraciones del lenguaje. Con todo, es necesario reconocer que cada vez es más frecuente y sistemática la aparición de investigaciones clínicas sistemáticas que apelan al análisis meramente estructural de algún fenómeno lingüístico. Ello ha permitido concluir que "[de] la naturaleza lingüística de los trastornos del lenguaje [...] se deduce que el comportamiento patológico es sistemático y de que en él se verifican ciertas hipótesis [...] Esta naturaleza lingüística de las manifestaciones patológicas opone la sistematicidad al azar" (MarcosOrtega, 1996, p. 164).

En este contexto, la perspectiva neuropsicológica luriana -en gran parte basada en la noción de sistema funcional complejo (Anojin, 1987)-supone un cambio sustancial en la forma de abordar las dificultades del lenguaje, ya que permite considerar que éste, como cualquier función psicológica, está integrado por una serie de mecanismos subyacentes que soportan las distintas operaciones de la actividad humana. Desde esta perspectiva, el trabajo del neuropsicólogo está dirigido -en primera instancia- a la cualificación del defecto primario y al análisis de sus mecanismos subyacentes (Quintanar-Rojas \& Solovieva, 2008; Solovieva, Torrado, Maravilla, \& Rivas, 2017). Lo anterior brinda -según Luria (1958)- tres ventajas fundamentales: (i) contribuye a una mayor exactitud en el diagnóstico, (ii) permite diferenciar entre condiciones que pueden ser similares externamente, pero que son esencialmente distintas, y (iii) proporciona las bases científicas y metodológicas adecuadas para generar métodos y vías de trabajo correctivo sistemático.

Así, para Luria \& Yudovich (1978), en el curso del desarrollo, afirman que las formas complejas de actividad se van integrando a nivel cerebral a partir de la operación conjunta de múltiples secciones corticales que aseguran su correcto funcionamiento. Así, para la realización de una tarea específica, se conforma un conjunto de zonas altamente especializadas, que se encuentran anatómicamente distantes entre sí, que se unen funcionalmente para dar soporte a una función determinada (Luria, 1979; Quintanar-Rojas \& Solovieva, 2008). El trabajo específico que realiza cada una de dichas zonas es denominado factor o mecanismo psicofisiológico (Akhutina, 2008; Xomskaya, 2002a, 2002b). En el caso del lenguaje, lo relevante es que, si se presenta algún daño o alteración neurológica, se verá afectado un factor psicofisiológico lo que, a su vez, provocará algún déficit en uno de los niveles del lenguaje. El análisis desde esta perspectiva se ha mostrado altamente eficiente pues se abandona el uso de términos como disfasia, dislalia, dislexia, disgrafía o retardo del lenguaje (Ardila, Rosselli, \& Matute, 2005; 
Azcoaga, 1990; Deus, Junqué, \& Vila, 1992; ManzanoLeón, Aguilera-Ruiz, Lozano-Segura, Yanicelli, \& Aguilar-Parra, 2017) porque se entiende que un mismo factor neuropsicológico puede dar lugar a distintas manifestaciones secundarias (Luria, 1959).

Para ilustrar el carácter idóneo del diagnóstico neurolingüístico frente a las categorías semiológicas tradicionales, se tomará en consideración la categoría de Trastorno Específico del Lenguaje (TEL) por ser la más reportada y extendida -aunque no libre de polémica-. En términos generales, el TEL se entiende como un déficit en la adquisición y desarrollo del lenguaje sin una causa neurológica, sensorial o física que lo justifique (Buiza, Rodríguez-Parra, \& Adrián, 2015). La prevalencia ha sido reportada cercana al $7 \%$ en niños monolingües y, con relación al género, de $6 \%$ en niñas y $8 \%$ en niños (Hincapié et al., 2007). Este trastorno se caracteriza por presentar problemas para el desarrollo natural y fluido del lenguaje, lo que interfiere, en gran medida, en la capacidad del niño para interactuar con el mundo, por lo que a futuro se perfilan como un grupo con menores oportunidades escolares y laborales (Hincapié et al., 2008). En este mismo sentido, los niños con TEL tienen un pobre rendimiento escolar y presentan problemas emocionales que dañan su desenvolvimiento social (Jackson-Maldonado \& Maldonado, 2017).

A pesar de lo anterior, desde hace unos años se ha cuestionado si este problema -en efecto- solo afecta al lenguaje, por lo que algunos autores reconocen las limitaciones de este término (Buiza et al., 2015). Dichas limitaciones se relacionan con la heterogeneidad de las manifestaciones clínicas que poseen los niños diagnosticados con TEL. También hay que agregar que los límites entre un trastorno del lenguaje y un retraso en su adquisición lingüística son difusos, hecho que genera grandes dificultades en la realización de un diagnóstico certero (Acosta, Moreno, \& Axpe, 2012). En este sentido, se sostiene que la perspectiva neuropsicológica de Luria permite superar estas dificultades al no partir de la identificación de síntomas en el niño para su diagnóstico, sino en su análisis cualitativo con el fin de conocer sus causas, factores o mecanismos psicofisiológicos subyacentes.

A propósito de lo anterior, es necesario considerar que autores como Jakobson (1966, 1971) han desarrollado un cuerpo teórico que vincula los factores neuropsicológicos propuestos por Luria con diversas manifestaciones lingüísticas, entre ellas, desórdenes fonológicos; sin embargo, se ha realizado a partir del estudio de las afasias. Así, este autor describe alteraciones fonológicas vinculadas a problemas de selección por similitud en casos de alteración de la discriminación fonológica entre segmentos con propiedades acústicas cercanas. También menciona dificultades en la combinación de rasgos articulatorios en casos de afectación de la integración propioceptiva de los órganos del habla y simplificaciones silábicas en los casos de afectación de la capacidad de combinación de segmentos de forma secuencial.

Respondiendo a esta limitación, en cuanto a la generalización de la asociación entre factores neurofisiológicos, neuropsicológicos y manifestaciones lingüísticas, se presenta un estudio de caso de un niño diagnosticado con TEL, que pretende mostrar cómo un diagnóstico certero donde se identifican las causas subyacentes al déficity un análisis lingüístico riguroso pueden ayudar a la 
identificación y análisis de las alteraciones del lenguaje, así como a la caracterización de los déficits fonológicos del niño. Además, se quiere mostrar cómo estos datos contribuyen al diseño de un programa de rehabilitación idóneo para cada tipo de paciente, ya que se atiende al déficit lingüístico específico que se presenta. Así, el objetivo es identificar los factores neurofisiológicos y neuropsicológicos alterados en un niño con problemas de lenguaje. A partir de esto, realizar un análisis sistemático de los errores fonológicos que se presentan para determinar la relación que existe entre ambos factores.

\section{Presentación del caso}

El paciente ME es un sujeto masculino de seis años y 10 meses de edad, hablante monolingüe de español, de lateralidad diestra. ME es llevado a la Unidad de Neuropsicología del Hospital Universitario de la ciudad de Puebla debido a que presenta dificultades en la producción de lenguaje oral, a saber: omisiones y sustituciones fonológicas, así como problemas en la articulación de ciertos fonemas. ME tiene dificultades en la motricidad gruesa y fina -necesarias para la precisión de cualquier acto dirigido a un objetivo- y en el movimiento fino y preciso de la lengua. Al momento de la evaluación, el niño se encontraba cursando el segundo grado de primaria y manifestaba una pobre socialización con pares debido a la falta de confianza en sí mismo como consecuencia de los déficits antes descritos. Como elemento importante, cabe señalar que el menor acudió a consulta con un diagnóstico previo de Trastorno Específico del Lenguaje con Compromiso de la Expresión Oral, otorgado en un servicio de Terapia del Lenguaje.

\section{Metodología}

El tipo de análisis que se llevó a cabo es de estudio de caso -de tipo descriptivo exploratorio- donde interesa, principalmente, realizar un análisis cualitativo de los déficits fonológicos presentes en el niño con problemas de lenguaje y su relación con las dificultades neurofisiológicas y neuropsicológicas. A continuación, se describen los procedimientos de evaluación realizados.

\section{Evaluación neurofisiológica}

Para la evaluación neurofisiológica se realizó un análisis electroencefalográfico en un equipo Nicolet Brain Lab con 19 electrodos colocados de acuerdo con el sistema 10-20 internacional: O1, O2, P3, P4, C3, C4, T3, T4, T5, T6, F1, F2, F3, F4, F7, F8, Pz, Cz y Fz. El electro-oculograma se registró en estado de reposo para excluir ensayos contaminados con movimientos oculares.

\section{Evaluación neuropsicológica}

La evaluación neuropsicológica se realizó en varias sesiones durante las cuales se utilizaron los siguientes instrumentos y sus respectivos apartados:

\section{Evaluación neuropsicológica infantil breve Puebla (Solovieva \& Quintanar-Rojas, 2017):}

- Regulación y control: dirigido a la evaluación de las funciones regulatorias de planeación y verificación de los lóbulos frontales. 
- Organización secuencial motora: exploración de áreas premotoras que permiten la organización del movimiento en el plano materializado, gráfico y del lenguaje.

- Integración cinestésica: evaluación de funciones táctiles y propioceptivas relacionadas con estructuras parietales secundarias.

- Integración fonemática o percepción selectiva y diferenciada de los sonidos del lenguaje asociada a porciones secundarias de los lóbulos temporales.

- Retención de información audioverbal y visual relacionada con estructuras diencefálicas, temporales y occipitales.

- Integración espacial o integración multimodal de sectores temporo-parieto-occipitales relacionados con la cognición espacial, el esquema corporal y la síntesis simultánea de estructuras lógico-gramaticales en el lenguaje.

- Activación inespecífica general asociada a estructuras frontoreticulares que permiten el adecuado establecimiento del nivel de alerta de la persona.

\section{Evaluación neuropsicológica de la actividad verbal} (Quintanar-Rojas \& Solovieva, 2010):

- Producción verbal oral.

- $\quad$ Lenguaje oral receptivo.

Evaluación clínica neuropsicológica de la afasia Puebla-Sevilla (Quintanar-Rojas, Solovieva, \& LeónCarrión, 2011):

- $\quad$ Organización Secuencial Motora.

- Integración Cinestésica.

- Integración Fonemática.
- Integración Espacial.

- Retención Visuo-Verbal.

- Retención Audio-Verbal.

- Organización Dinámica.

\section{Evaluación neuropsicológica de la actividad escolar (Solovieva \& Quintanar-Rojas, 2012):}

- Evaluación neuropsicológica del Éxito Escolar.

- Evaluación Neuropsicológica de la Integración Espacial.

- Evaluación Neuropsicológica de la Actividad Mnésica.

\section{Evaluación lingüística}

Una vez realizada la evaluación neuropsicológica y neurofisiológica, se realizaron a cabo dos tareas específicas para la obtención de los datos lingüísticos. En la primera tarea se le presentaron al menor dos caricaturas animadas sin diálogos (Raimbaud, 1998). Cada video tenía una duración aproximada de seis minutos. Después de verlas, se le solicitó recontar los hechos observados en ellas. La segunda tarea consistió en una entrevista semi-estructurada, en la cual se realizaron preguntas sobre sus gustos e intereses, así como de las actividades que lleva a cabo durante el día. Ambas tareas fueron grabadas con la autorización expresa de los padres, a quienes se les explicó sobre la investigación y se les informó sobre el manejo ético que se les daría a los datos. Así mismo se dejó en claro que el niño podría abandonar el estudio en el momento que lo deseara.

Para el tratamiento informático de los datos empleamos el programa ELAN (Sloetjes \& Wittenburg, 2008) y para su transcripción adoptamos el sistema de 
etiquetado propuesto por el Grupo Val.Es.Co (Briz \& Grupo Val.Es.Co, 2002). Finalmente, en aquellos fragmentos donde se presentaban alteraciones articulatorias se efectuó una transcripción fonológica. Posteriormente, se realizó la búsqueda de las palabras y los segmentos fonológicos que presentaban errores en la articulación y los agrupamos de acuerdo con el tipo de error.

\section{Análisis y resultados}

El análisis neurofisiológico reveló desviaciones locales de la actividad cerebral en forma de grupos de ondas de rango Theta en la región parietal posterior del hemisferio derecho de origen profundo, lo cual se encuentra en estrecha correspondencia con los hallazgos neuropsicológicos que se describen a continuación.

En la evaluación neuropsicológica se encontraron dificultades en dos aspectos específicos: (i) en el mecanismo de integración cinestésica y (ii) en la organización secuencial de movimientos. El primer mecanismo está relacionado con estructuras corticales parietales, que permiten integrar adecuadamente información propioceptiva, por lo que el menor presenta dificultades para determinar la posición de los miembros de su cuerpo cuando se le impide utilizar la visión para modular el movimiento. De este modo, se puede apreciar que la actividad atípica encontrada en el análisis neurofisiológico presenta una relación estrecha con elementos neuropsicológicos en la actividad del niño.

\footnotetext{
${ }^{1}$ Las dificultades descritas no son secundarias a una alteración en la sensibilidad o motricidad; el menor reconoce puntos de contacto en la piel y la lengua, así como grafestesias dibujadas sobre sus manos y mejillas, y no se observaron signos de parálisis o paresias.
}

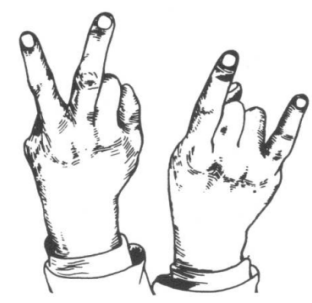

Figura 1. Tarea de posiciones de dedos tomado de Luria (2005, p. 414). Se solicita al paciente cerrar los ojos mientras se colocan los dedos en la posición indicada en la imagen; posteriormente se le pide que reproduzca dichas posiciones sin utilizar la visión.

Este déficit se manifiesta, en el plano corporal, en errores en la reproducción de posiciones de los dedos en ambas manos (figura 1), tarea en la cual el niño realiza movimientos aleatorios y refiere no saber en qué posición tiene sus dedos; así mismo. En el plano articulatorio del lenguaje, se evidencia ausencia de precisión en los movimientos de la lengua y se observan sustituciones fonológicas entre segmentos cercanos en rasgos articulatorios ante tareas de lectura, escritura, repetición y habla espontánea1.

En cuanto a la organización secuencial de movimientos y acciones, se hace referencia a la capacidad de encadenar de forma fluida y organizada series de movimientos dirigidos a un fin determinado.
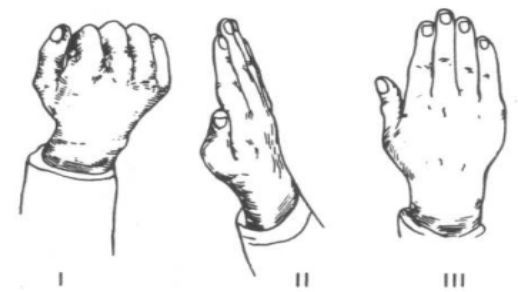

Figura 2. Tarea de secuencia motora manual Puño-FiloPalma tomado de Luria (2005, p. 422). Se solicita al paciente que realice la secuencia de movimientos arriba indicados con cada una de sus manos. 
Así, cuando se le solicitaba al niño realizar cadenas de movimientos presentaba dificultades para efectuarlo de forma fluida, completa o en el orden adecuado, incurriendo en simplificaciones ${ }^{2}$ (figura 2 ). Esto también repercutía fundamentalmente en la pronunciación de palabras largas (/paldamas/ por /palmadas/), sílabas trabadas (/kus/ por /krus/), diptongos (/xualua/ por /xaula/) y pares de palabras similares en su composición (/tropa/-/tropo/ por /tropa/-/potro/). En resumen, estos dos mecanismos presentan un desarrollo insuficiente en el menor, lo que provoca no sólo dificultades en la producción oral, sino también en la lecto-escritura ${ }^{3}$.

Ahora bien, la producción lingüística del niño estaba compuesta por una gran cantidad de errores fonológicos, principalmente omisiones y sustituciones que a priori podrían interpretarse como fenómenos de simplificación fonológica, característicos en el desarrollo de la adquisición del lenguaje. No obstante, podría ser una explicación simplista porque sólo se estaría describiendo el tipo de error sin explicar cuál o cuáles pueden ser las causas que subyacen a ellos. Así pues, en la Tabla 1 se presentan los errores en la producción lingüística del niño agrupados por clase e incidencia.
Tabla 1

Tipo e incidencia de errores fonológicos de ME.

\begin{tabular}{lcc}
\hline Tipo de error & Subtipo & Frecuencia \\
\hline Asimilación & Progresiva & 3 \\
Sustitución & Pegresiva & 5 \\
& Modo & 10 \\
Omisión & Silábica & 25 \\
& Aféresis & 16 \\
Inserción & Síncopa & 35 \\
& Apócope & 26 \\
& Prótesis & 34 \\
Total & Epéntesis & 0 \\
& Paragoge & 3 \\
\cline { 2 - 3 } & & 159 \\
\hline
\end{tabular}

Se presentan nueve ejemplos de este fenómeno, cinco regresivos -el fonema desviado hereda uno o más rasgos de un segmento subsecuente- y tres progresivos -el fonema desviado hereda uno o más rasgos de un segmento previo.

\section{Asimilación}

En (1) y (2), se exponen ejemplos de errores de asimilación regresiva y progresiva, respectivamente:
(1) a./kuraratfa/ > /kukaratfa/
b./pote/ > /bote/
(2) a./kareras/ > /kareras/
b./amararon/ > /amararon/

\footnotetext{
2 Por ejemplo, en series de movimientos manuales como puño-filopalma o de coordinación recíproca de ambas manos.

${ }^{3}$ En la lectoescritura se observaron dificultades análogas a las descritas en el plano oral; por ejemplo, ante la escritura de oraciones el niño comete errores como escribir "compor" en lugar de "compró" y en la lectura, se observan sustituciones entre fonemas oclusivos como /b/ y /t/.
} 
En (1a), existe un proceso anticipatorio por el cual /r/ presenta una asimilación total de los rasgos del segmento que le sigue; mientras que en (1b), la oclusiva bilabial sonora /b/ se ensordece por influencia de la consonante dental sorda /t/ que le sigue. Por su parte, en (2a) y (2b), se puede observar que el rasgo [+trill] de la vibrante múltiple de la sílaba tónica se extiende a la sílaba siguiente, lo que puede ser causado por la dificultad en la inhibición del programa motor -una vez que se ha producido-. Lo anterior debido a la inadecuada aferentación de retorno, que impide la valoración del resultado de la acción y, por tanto, lleva a la persistencia de dicho rasgo ${ }^{4}$. Así pues, en primera instancia, los procesos de asimilación pueden ser atribuidos a las dificultades que presenta el niño con la organización secuencial del movimiento y, como ya se dijo, su relación con la retroalimentación propioceptiva de los órganos fonoarticuladores.

\section{Sustituciones}

Las sustituciones fueron frecuentes en el habla del niño. Para su análisis, las dividimos en tres: (i) punto de articulación, (ii) modo de articulación y (iii) sílaba. En el primer caso, dicho fenómeno se presentó en diez ocasiones, en fonemas que compartían modo de articulación e, invariablemente, el niño atrasaba el punto de articulación, como se muestra en los ejemplos de (3):

\footnotetext{
4 De este modo, se puede apreciar una estrecha relación entre los errores fonológicos del niño y los factores neuropsicológicos descritos. La alteración en la integración de información propioceptiva dificulta los adecuados procesos de retroalimentación necesarios para el movimiento preciso y lleva secundariamente a problemas en el carácter secuencial de los segmentos del habla.

${ }^{5}$ Se asumen como errores fonológicos y no morfosintácticos o léxicos, debido a que cuando se pregunta al niño ¿̇a quién?, señala al personaje de la caricatura indicado a pesar de que su producción no corresponda a este.
}

$$
\begin{aligned}
& \text { (3) a./xueron/ > /fueron/ } \\
& \text { b. /xue/ > /fue/ }
\end{aligned}
$$

Así, en ambos casos, la fricativa labiodental /f/ es producida como un fricativa velar /x/. Además, si atendemos a que este error ocurre antes de diptongo -encabezado por un vocal posterior- podemos asumir que el cambio de punto de articulación responde a una simplificación del patrón motor, ya que están más cercanas articulatoriamente / $\mathrm{x} / \mathrm{y} / \mathrm{u} /$ que /f/ y /u/. En este caso en particular, es necesario mencionar que no se trata de una variante dialectal, lo cual se hace evidente al contrastar la producción del niño con la de sus hermanas y padres, quienes no cometen este tipo de sustitución.

En el caso de las sustituciones por modo de articulación, se presentó el patrón contrario: los fonemas desviados compartían el punto de articulación, dentoalveolar. Así, se presentaron sustituciones entre los fonemas /t/, /d/, /l/, /r/ y /s/ como se muestra en (4):

$$
\begin{aligned}
(4)^{5} \text { a./pegarte/ } & >\text { /pegarle/ } \\
\text { b. } / \text { te/ } & >/ \text { se/ } \\
\text { c. } / \text { de/ } & >/ \text { se/ } \\
\text { d. } / \text { le/ } & >/ \mathrm{se} /
\end{aligned}
$$

Finalmente, en el caso de las sustituciones silábicas, como vemos en (5), se presentaron como simplificaciones de la estructura de sílaba trabada en 
posición inicial. Resulta importante señalar que este fenómeno no se presentó en posición intermedia o a final de palabra:

$$
\begin{aligned}
& \text { (5) a./upues/ }>\text { /despues/ } \\
& \text { b./opues/ }>\text { /despues/ } \\
& \text { c./mpues/ }>\text { /después/ }
\end{aligned}
$$

\section{Omisiones}

Como en el caso anterior, este fenómeno fue bastante frecuente. Este tipo de errores se clasifican de acuerdo con la posición en la que se produce la omisión. Así, si se presenta a inicio de palabra se le llama aféresis; a interior, síncopa y al final, apócope. En el primer caso, los errores tendían a presentar la elisión de la sílaba inicial, compuesta por vocal, en palabras con tres o más sílabas. Además, en todos los casos, ocurrió cuando la vocal elidida antecedía a consonantes obstruyentes -oclusivas, fricativas y africadas:

$$
\begin{aligned}
& \text { (6) } / \text { kabo/ }>\text { /akabo/ } \\
& \text { b./kuerdo/ }>\text { /akuerdo/ } \\
& \text { c./simos/ }>\text { /isimos/ } \\
& \text { d./tgaron/ }>\text { /etaron/ }
\end{aligned}
$$

Por su parte, en cuanto a los casos de síncopa, estos dieron como resultado la simplificación de la estructura silábica, de CCV a CV y de CVC a CV. Como

\footnotetext{
${ }^{6}$ Se retoman como errores fonológicos y no morfosintácticos, dado que, dentro de la producción del niño, se encuentran presentes otros elementos que dan cuenta de la flexión por número, siendo los elementos señalados las excepciones que no se explican por una dificultad en la determinación de esta propiedad. Por ejemplo, en el ejemplo 8b, la producción del niño fue: "Te $t(r) a t a b a$ que los gatos, o ¿qué? - ¿qué era(n)?"; donde el artículo y el sustantivo poseen una adecuada flexión morfosintáctica, que no corresponde con el verbo que se encuentra en la posición final.
}

es esperable, los problemas se presentaron principalmente en los fonemas /I/ y /r/. Lo anterior se encuentra en estrecha relación con la alteración de la organización secuencial de los movimientos, dado que resulta más sencillo para el niño integrar cadenas de dos segmentos que aquellas compuestas por tres. Finalmente, los casos de apócope también fueron frecuentes y todos los errores se presentaron en fonemas dentoalveolares, cuya producción requiere de una aferentación propioceptiva precisa de la lengua. En (7) y (8) presentamos ejemplos de síncopa y apócope, respectivamente:

$\begin{aligned} \text { a./goserías/ } & >\text { /groserías/ } \\ \text { b./sabo/ } & >\text { /salbo/ } \\ \text { c./síkulo/ } & >\text { /sírkulo/ } \\ \text { d./pitfaron/ } & >\text { /pintfaron/ } \\ (8)^{6} \text { a./la/ } & >\text { /las/ } \\ \text { b./era/ } & >\text { /eran/ } \\ \text { c. } / \text { niño/ } & >\text { /niños/ } \\ \text { d. } / \text { fina/ } & >\text { /final/ }\end{aligned}$

\section{Inserciones}

En el caso de las inserciones, los errores se presentaron en los mismos fonemas dentoalveolares, lo que refuerza la hipótesis de que existe una dificultad en el análisis y síntesis de la información propioceptiva-cinestésica del aparato 
fonoarticulador, lo que interfiere con la precisión del movimiento. En (9) presentamos ejemplos de esto:

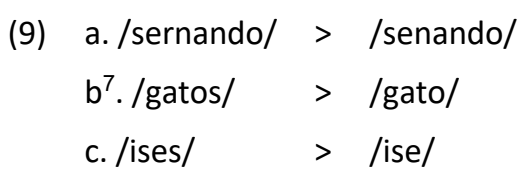

\section{Discusión}

Para ponderar los resultados de este trabajo es necesario destacar que: (i) el lenguaje es, en gran parte, un acto motor, puesto que requiere el control y coordinación de múltiples órganos para la producción selectiva de los sonidos de un sistema dado (Zhinkin, 1960) y (ii) al igual que toda función psicológica superior, el lenguaje, incluido su componente motor, se desarrolla a lo largo de la ontogenia y requiere como elemento fundamental un sistema nervioso sano y un entorno sociocultural propicio para su formación (Luria \& Tsvetkova, 1966; Luria \& Yudovich, 1978). En el caso expuesto, el análisis neurofisiológico reveló la existencia de actividad eléctrica atípica en territorio parietal posterior derecho de origen profundo, lo que se relaciona en el nivel neuropsicológico con un déficit funcional en los mecanismos de integración de información propioceptiva y de la secuenciación del movimiento, por lo que representan un obstáculo para el adecuado desarrollo del lenguaje.

Bernstein (2006a, 2006b, 2006c) afirma que un componente fundamental para la precisión del acto motor y el desarrollo de estereotipos dinámicos programas automatizados de movimiento- es la

\footnotetext{
${ }^{7}$ Nuevamente, se asume como error fonológico y no morfosintáctico dado su contexto de producción ("Era como un gatos").
}

aferentación de retorno, es decir, el continuo flujo de información acerca de la posición y estado de aquellos órganos que serán inervados para dar origen al movimiento. Es justamente en este componente en el que se observan dificultades en el menor, lo que repercute negativamente en el desarrollo del lenguaje.

Como menciona Jakobson (1968), en todo sistema lingüístico lo que importa no son los sonidos aislados per se, sino las distinciones que se establecen entre los distintos sonidos dentro del sistema fonológico de cada lengua. Evidencia de esto es el proceso de desarrollo lingüístico en el que la progresión de adquisición de fonemas por el niño responde al establecimiento de relaciones contrastivas entre distintas unidades (i.e. vocal-consonante, oral-nasal, etcétera). A partir de dichas distinciones, el sistema fonológico se estructura jerárquicamente, tal y como afirman Lecours \& Lhermitte (1969), de manera que desde la combinación de un número determinado de rasgos se forman los fonemas de la lengua, los cuales dan lugar a morfemas y estos a sintagmas. Sin embargo, ante un déficit en el factor neuropsicológico que permite la integración de la información propioceptiva, como el que ocurre en este caso, la diferenciación entre los elementos dentro del sistema lingüístico se dificulta, sobre todo entre elementos cercanos por sus rasgos articulatorios. Lo anterior se hace evidente si se analiza la distancia paradigmática que existe entre los distintos fonemas del sistema lingüístico del español mexicano en cuanto a sus rasgos constituyentes (Figura 3) y lo contrastamos con la ejecución del paciente. 
Tabla 2

Rasgos de los fonemas del español mexicano. Realizado a partir de (Hayes, 2009).

\begin{tabular}{|c|c|c|c|c|c|c|c|c|c|c|c|c|c|c|c|c|c|}
\hline Fonema & $/ p /$ & $/ \mathrm{b} /$ & $/ \mathrm{m} /$ & $/ f /$ & $/ \mathrm{t} /$ & $/ d /$ & /s/ & $/ \mathrm{n} /$ & /I/ & $/ r /$ & $/ r /$ & $/ \mathrm{t} /$ & $/ \mathrm{d} /$ & $/ \mathrm{n} /$ & $/ \mathrm{k} /$ & $/ g /$ & $|x|$ \\
\hline Sonorante & - & - & + & - & - & - & - & + & + & + & + & - & - & + & - & - & - \\
\hline Continuante & - & - & - & + & - & - & + & - & + & + & + & - & + & - & - & - & + \\
\hline Liberación ret. & - & - & 0 & + & - & - & + & 0 & 0 & 0 & 0 & + & + & 0 & - & - & + \\
\hline Aproximante & - & - & - & - & - & - & - & - & + & + & + & - & - & - & - & - & - \\
\hline Tap & - & - & - & - & - & - & - & - & - & + & - & - & - & - & - & - & - \\
\hline Trill & - & - & - & - & - & - & - & - & - & - & + & - & - & - & - & - & - \\
\hline Nasal & - & - & + & - & - & - & - & + & - & - & - & - & - & + & - & - & - \\
\hline Sonora & - & + & + & - & - & + & - & + & + & + & + & - & + & + & - & + & - \\
\hline Labial & + & + & + & + & - & - & - & - & - & - & - & - & - & - & - & - & - \\
\hline Laiodental & - & - & - & + & - & - & - & - & - & - & - & - & - & - & - & - & - \\
\hline Coronal & - & - & - & - & + & + & + & + & + & + & + & + & + & + & - & - & - \\
\hline Anterior & 0 & 0 & 0 & 0 & + & + & + & + & + & + & + & - & - & - & - & - & - \\
\hline Distribuida & 0 & 0 & 0 & 0 & - & - & - & - & - & - & - & + & + & - & - & - & - \\
\hline Estridente & 0 & 0 & 0 & 0 & - & - & + & - & - & - & - & + & + & - & - & - & - \\
\hline Lateral & - & - & - & - & - & - & - & - & + & - & - & - & - & - & - & - & - \\
\hline Dorsal & - & - & - & - & - & - & - & - & - & - & - & - & - & - & + & + & + \\
\hline
\end{tabular}

Así, utilizando un análisis de correspondencia múltiple de dichos rasgos para generar clusters de agrupamiento, se pudieron distinguir cuatro grupos que presentaban mayor homogeneidad entre sí (Figura 3): fonemas anteriores con participación labial -en rojo-, fonemas que requieren del uso de la lengua y que se articulan en porciones dentoalveolares o palatales como en el caso de la /n/ -en azul-, fonemas centro-posteriores que comparten características obstruyentes y de liberación retardada -en amarilloy fonemas velares -en morado-. Adicionalmente, se señaló con color verde la distribución de los fonemas /d/ y /t/ que, por su configuración de rasgos, comparten características con múltiples elementos dentro del sistema. Finalmente, en amarillo se señalizaron unidades que pueden caracterizarse a grandes rasgos como fonemas posteriores. 


\begin{tabular}{|c|c|c|c|c|c|c|c|c|c|c|c|c|c|c|c|c|c|}
\hline /p/ & 16 & 15 & 12 & 13 & 11 & 10 & 5 & 5 & 5 & 7 & 7 & 9 & 10 & 8 & 9 & 11 & 10 \\
\hline b/ & 15 & 16 & 13 & 12 & $1 \underline{0}$ & 11 & 6 & 6 & 6 & 8 & 8 & 8 & 9 & 9 & 8 & 10 & 11 \\
\hline $\mathrm{n} /$ & 12 & 13 & 16 & 10 & 7 & 8 & 7 & 7 & 7 & 11 & 11 & 6 & 7 & 7 & 6 & 7 & 8 \\
\hline /f/ & 13 & 12 & 10 & 16 & 8 & 7 & 5 & 5 & 5 & 5 & 5 & 10 & 9 & 9 & 10 & 8 & \\
\hline$/ \mathrm{t} /$ & 11 & 10 & 7 & 8 & 16 & 15 & 10 & 10 & 10 & 12 & 11 & 13 & 12 & 10 & 11 & 13 & 12 \\
\hline /d/ & 10 & in & 8 & 7 & 15 & 16 & 11 & 11 & 11 & 13 & 12 & 12 & 11 & 11 & 10 & 12 & 13 \\
\hline /1/ & 5 & 6 & 7 & 5 & 10 & 11 & 16 & 14 & 14 & 12 & 11 & 10 & 7 & 9 & 8 & 7 & 8 \\
\hline$/ \mathbf{r} /$ & 5 & 6 & 7 & 5 & 10 & 11 & 14 & 16 & 14 & 12 & 11 & 10 & 7 & 9 & 8 & 7 & 8 \\
\hline$/ \mathrm{r} /$ & 5 & 6 & 7 & 5 & 10 & 11 & 14 & 14 & 16 & 12 & 11 & 10 & 7 & 9 & 8 & 7 & 8 \\
\hline$/ \mathrm{n} /$ & 7 & 8 & 11 & 5 & 12 & 13 & 12 & 12 & 12 & 16 & 15 & 10 & 9 & 9 & 8 & 9 & 10 \\
\hline$/ \mathrm{n}$ & 7 & 8 & 11 & 5 & 11 & 12 & 11 & 11 & 11 & 15 & 16 & 9 & 10 & 10 & 9 & 10 & 11 \\
\hline$/ \mathrm{s}$ & 9 & 8 & 6 & 10 & 13 & 12 & 10 & 10 & 10 & 10 & 9 & 16 & 13 & 13 & 12 & 10 & 0 \\
\hline$/ t /$ & 10 & 9 & 7 & 9 & 12 & 11 & 7 & 7 & 7 & 9 & 10 & 13 & 16 & 14 & 11 & 11 & 10 \\
\hline$/ \mathrm{j} /$ & 8 & 9 & 7 & 9 & 10 & 11 & 9 & 9 & 9 & 9 & 10 & 13 & 14 & 16 & 11 & 9 & 10 \\
\hline$/ x /$ & 9 & 8 & 6 & 10 & 11 & 10 & 8 & 8 & 8 & 8 & 9 & 12 & 11 & 11 & 16 & 14 & 13 \\
\hline$/ \mathrm{k} /$ & 11 & 10 & 7 & 8 & 13 & 12 & 7 & 7 & 7 & 9 & 10 & 12 & 11 & 9 & 14 & 16 & 15 \\
\hline /g/ & 10 & 11 & 8 & 7 & 12 & 13 & 8 & 8 & 8 & 10 & 11 & 13 & 10 & 10 & 13 & 15 & 16 \\
\hline
\end{tabular}

Figura 3. Matriz de relaciones de similitud entre las unidades fonológicas del español. Los números representan el número de rasgos compartidos entre cada par de fonemas señalados.

Lo anterior se puede relacionar con las observaciones de Jakobson (1968), quien afirma que es más fácil identificar relaciones contrastivas entre distintas clases de percepciones, que entre percepciones dentro de una misma clase. En el caso del paciente descrito, las alteraciones se presentan principalmente ante elementos de una misma clase; por ejemplo, en la sustitución de "pote" por "bote", los fonemas /p/ y /b/ pertenecen al grupo previamente señalado en color rojo. Asimismo, los errores en los ejemplos (1a), (2), (4), (7), (8) y (9) se relacionan con el grupo señalado en color azul. Al respecto de estos últimos, cabe señalar que constituyen un grupo altamente homogéneo según el análisis realizado. Esto junto con el hecho de que el paciente presenta dificultades particulares en el factor de integración de información propioceptiva de la lengua, provocan un déficit selectivo en la selección de elementos dentro de este grupo. Lo anterior se vuelve más evidente cuando se recuerda que los fonemas dentoalveolares -en los que se presentan más alteraciones- constituyen el paradigma más amplio en español (8 fonemas), por lo que el niño debe realizar distinciones mucho más finas cuando articula este tipo de sonidos.

La identificación de las causas y características propias del cuadro del paciente resultan de gran importancia para la planeación y ejecución de programas de intervención adecuados. Si se partiera del diagnóstico inicial de TEL con compromiso de la expresión oral, el rango de objetivos terapéuticos posibles se hace demasiado extenso. Ello debido a que podría considerar desde aspectos motores de la articulación hasta elementos metacognitivos involucrados en la expresión de ideas en el discurso. Sin embargo, a partir del análisis neurolingüístico, se 
torna evidente la necesidad de proporcionar medios para suplir las deficiencias en la aferentación de retorno que impide la adecuada diferenciación entre los elementos de una misma clase. Asimismo, resalta la importancia de enfatizar en las características distintivas de la articulación de los fonemas dentoalveolares. Ahora bien, es necesario tener en cuenta que la matriz de similitud de rasgos descrita se formó a partir de la premisa de que todos tenían un mismo peso en las relaciones entre los fonemas, sin embargo, este puede no ser el caso. En este sentido, se deben realizar estudios para determinar el peso de cada uno de los rasgos en la estructuración paradigmática del sistema fonológico del español, lo cual debe responder a los procesos de oposición a través de los cuales el niño va formando dicha jerarquización.

Finalmente, llama la atención el hecho de que actividad eléctrica atípica se presentara en estructuras del hemisferio derecho. Esto es así debido a que comúnmente se asocia al lenguaje con el hemisferio izquierdo, sobre todo en sujetos sanos diestros, quienes en un $\mathbf{9 2 . 5 \%}$ de los casos presentan lateralización de esta función en dicho hemisferio (Knecht et al., 2000). También resulta interesante que el origen de la actividad eléctrica atípica se sospeche en estructuras profundas, lo cual se relaciona con los postulados de Vigotsky (2016) sobre la estructuración jerárquica del sistema nervioso y la importancia de las estructuras subcorticales en las etapas tempranas del desarrollo. Esto llevaría a pensar acerca de si en el caso presente existió una alteración de estructuras subcorticales que, secundariamente, produjo una alteración en el desarrollo funcional de las porciones parietales de la corteza cerebral. En las últimas décadas, el interés en la participación de las estructuras subcorticales en el lenguaje ha llevado al descubrimiento de alteraciones articulatorias, sintácticas y semánticas asociadas a éstas (Lieberman, 2002; Radanovic \& Scaff, 2003), aunque aún es necesaria mayor investigación.

\section{Conclusiones}

El análisis neuropsicológico, neurofisiológico y lingüístico ayudaron a determinar como causas del déficit del paciente un desarrollo insuficiente de los mecanismos de integración de información propioceptiva y de organización secuencial de movimientos, asociado esto a una actividad atípica en estructuras parietales profundas del hemisferio derecho. La alteración de las bases fundamentales del movimiento voluntario tuvo como consecuencia el desarrollo inadecuado del sistema fonológico del niño, de forma tal que se presentan múltiples errores asociados a la dificultad para diferenciar entre percepciones propioceptivas cercanas entre sí. El análisis lingüístico permitió evidenciar un compromiso en la selección de las unidades fonémicas, que se relaciona directamente con el nivel de similitud entre sus rasgos constituyentes. Asimismo, este análisis contribuyó a puntualizar que la alteración se focaliza en los fonemas dentoalveolares, puesto que su articulación requiere una adecuada aferentación propioceptiva de la lengua. Esto se debe a que esta clase de fonemas es el paradigma más amplio en español y la desviación de un solo rasgo provocaría la modificación completa del segmento, considérese, por ejemplo, el caso de las líquidas /r/, /r/ y /l/. 
Lo anterior respalda el hecho de que el lenguaje se comporta como un sistema altamente organizado y jerarquizado en el que es posible descubrir las relaciones existentes entre sus múltiples niveles. EI análisis de los rasgos que conforman los fonemas de la lengua es un procedimiento útil para dilucidar dicha estructura y explicar la sistematicidad de los síntomas lingüísticos en casos de patología o desarrollo atípico del lenguaje. Finalmente, los datos neurofisiológicos aportan información importante sobre la participación de las estructuras subcorticales del hemisferio derecho en la integración de la información somato sensorial como base de la articulación del lenguaje, llamando la atención sobre la necesidad de investigar a profundidad sobre esto.

\section{Referencias}

Acosta, V., Moreno, A., \& Axpe, M. (2012). Implicaciones clínicas del diagnóstico diferencial temprano entre Retraso de Lenguaje ( $R L$ ) y Trastorno Específico del Lenguaje (TEL). Universitas Psychologica, 11(1), 279-291. https://doi.org/10.11144/Javeriana.upsy11-1.icdd

Akhutina, T. (2008). Neuropsicología de la edad escolar: Una aproximación histórico-cultural. Acta Neurológica Colombiana, 24(2), S17-S30.

Anojin, P. K. (1987). Psicología y la filosofía de la ciencia: Metodología del sistema funcional. México: Trillas.

Ardila, A., Rosselli, M., \& Matute, E. (2005). Neuropsicología de los trastornos del aprendizaje. México: Manual Moderno.

Azcoaga, J. E. (1990). Los retardos del lenguaje en el niño. Buenos Aires: Editorial Paidós.

Bernstein, N. (2006a). Basic Methodological Positions of the Physiology of Movements. Journal of Russian \& East European Psychology, 44(2), 12-32. https://doi.org/10.2753/RPO1061-0405440201

Bernstein, N. (2006b). From Reflex to Model of the Future. Journal of Russian \& East European Psychology, 44(2), 93-
98. https://doi.org/10.2753/RPO1061-0405440205

Bernstein, N. (2006c). Paths and Tasks of the Physiology of Activeness: Essay No. 11. Journal of Russian \& East European Psychology, 44(2), 33-59. https://doi.org/10.2753/RPO1061-0405440202

Briz, A., \& Grupo Val.Es.Co. (2002). La transcripción de la lengua hablada: El sistema del grupo Val.Es.Co. Español Actual: Revista de Español Vivo, (77), 57-86. Recuperado de https://www.researchgate.net/publication/331676841_La _transcripcion_de_la_lengua_hablada_el_sistema_del_gru po_ValEsCo

Buiza, J. J., Rodríguez-Parra, M. J., \& Adrián, J. A. (2015). Trastorno Específico del Lenguaje: Marcadores psicolingüísticos en semántica y pragmática en niños españoles. Anales de Psicología, 31(3), 879-889. https://doi.org/10.6018/analesps.31.3.180091

Deus, J., Junqué, C., \& Vila, M. (1992). Disfasia pura: Un estudio neuropsicológico y de neuroimagen. Revista de Logopedia, Foniatría y Audiología, 12(4), 231-242. https://doi.org/10.1016/S0214-4603(92)75553-3

Hayes, B. (2009). Introductory Phonology. West Sussex: Wiley-Blackwell.

Hincapié, L., Giraldo, M., Castro, R., Lopera, F., Pineda, D., \& Lopera, E. (2007). Propiedades lingüísticas de los trastornos específicos del desarrollo del lenguaje. Revista Latinoamericana de Psicología, 39(1), 47-61. https://doi.org/10.14349/rlp.v39i1.569

Hincapié, L., Giraldo, M., Lopera, F., Pineda, D., Castro, R., Lopera, J. P., ... Arboleda, A. (2008). Trastorno Específico del Desarrollo del Lenguaje en una población infantil colombiana. Universitas Psychologica, 7(2), 557-569. Recuperado de https://revistas.javeriana.edu.co/index.php/revPsycho/arti cle/view/557

Jackson-Maldonado, D., \& Maldonado, R. (2017). Grammaticality differences between Spanish-speaking children with specific language impairment and their typically developing peers. International Journal of Language \& Communication Disorders, 52(6), 750-765. https://doi.org/10.1111/1460-6984.12312

Jakobson, R. (1966). Linguistic types of aphasia. En C. Carterette (Ed.), III Speech Language and Communication: Forum in Medical Sciences (pp. 307-333). Los Angeles: University of California Los Angeles. 
Jakobson, R. (1968). Child Language, Aphasia and Phonological Universals. Netherlands: Mouton Publishers.

Jakobson, R. (1971). Selected writings II: Word and language. The Hague: Mouton \& Co.

Knecht, S., Deppe, M., Dräger, B., Bobe, L., Lohmann, H., Ringelstein, E., \& Henningsen, H. (2000). Language lateralization in healthy right-handers. Brain: A Journal of Neurology, $123 \quad\left(\begin{array}{ccc}\text { Pt } & 1),\end{array}\right.$ https://doi.org/10.1093/brain/123.1.74

Lecours, A. R., \& Lhermitte, F. (1969). Phonemic paraphasias: Linguistic structures and tentative hypothesis. Cortex; a Journal Devoted to the Study of the Nervous System and Behavior, 5(3), 193-228. https://doi.org/10.1016/s0010-9452(69)80031-6

Lieberman, P. (2002). Human language and our reptilian brain: The subcortical bases of speech, syntax, and thought. Cambridge: Harvard University Press.

Luria, A. R. (1958). Dynamic Approach to the Mental Development of the Abnormal Child. Journal of Intellectual Disability Research, 2(2), 37-52. https://doi.org/10.1111/j.1365-2788.1958.tb00384.x

Luria, A. R. (1959). Experimental study of the higher nervous activity of the abnormal child. Journal of Mental Deficiency Research, 3, 1-22.

Luria, A. R. (1979). El cerebro en acción (2da ed.). Barcelona, España: Fontanella.

Luria, A. R. (2005). Las funciones corticales superiores del hombre. México: Distribuciones Fontamara.

Luria, A. R., \& Tsvetkova, L. S. (1966). Rehabilitative Education and Its Importance for Psychology and Pedagogy. Soviet Review, 7(2), 3-12. https://doi.org/10.2753/RSS1061-142807023

Luria, A. R., \& Yudovich, F. (1978). The role of language on the development of mental processes. Infancia $y$ Aprendizaje, 1(3), 5-18. https://doi.org/10.1080/02103702.1978.10821701

Manzano-León, A., Aguilera-Ruiz, C., Lozano-Segura, M. C., Yanicelli, C. C., \& Aguilar-Parra, J. M. (2017). Conectivismo y dislexia. International Journal of Developmental and Educational Psychology. Revista INFAD de Psicología., 4(1), 253-260.

https://doi.org/10.17060/ijodaep.2017.n1.v4.1054
Marcos-Ortega, J. (1996). Roman Jakobson precursor de la neuropsicología cognitiva. En S. Cuevas \& J. Haidar (Eds.), La imaginación y la inteligencia en el lenguaje: Homenaje a Roman Jakobson (pp. 161-176). México: Instituto Nacional de Antropología e Historia.

Quintanar-Rojas, L, \& Solovieva, Y. (2008). Aproximación histórico-cultural: Fundamentos teórico-metodológicos. En J. Eslava, L. Mejía, L. Quintanar, \& Y. Solovieva (Eds.), Los trastornos del aprendizaje: Perspectivas neuropsicológicas (pp. 145-181). Colombia: Magisterio.

Quintanar-Rojas, L, Solovieva, Y., \& León-Carrión, J. (2011). Evaluación clínico neuropsicológica de la afasia PueblaSevilla. Puebla: Benemérita Universidad Autónoma de Puebla.

Quintanar-Rojas, Luis, \& Solovieva, Y. (2010). Evaluación neuropsicológica de la actividad verbal. Puebla: Benemérita Universidad Autónoma de Puebla.

Radanovic, M., \& Scaff, M. (2003). Speech and language disturbances due to subcortical lesions. Brain and Language, 84(3), 337-352. https://doi.org/10.1016/s0093$934 \times(02) 00554-0$

Raimbaud, J. (1998). Oggy and the cockroaches. Francia: Xilam.

Sloetjes, H., \& Wittenburg, P. (2008). ELAN and ISO DCR. Presentado en Proceedings of the 6th International Conference on Language Resources and Evaluation.

Solovieva, Y., \& Quintanar-Rojas, L. (2012). Evaluación neuropsicológica de la actividad escolar. Puebla, México: Benemérita Universidad Autónoma de Puebla.

Solovieva, Y., \& Quintanar-Rojas, L. (2017). Evaluación neuropsicológica infantil breve-Puebla. Puebla, México: Benemérita Universidad Autónoma de Puebla.

Solovieva, Y., Torrado, O., Maravilla, L., \& Rivas, X. (2017). Análisis neuropsicológico diferencial en dos casos diagnosticados con TDAH. Informes Psicológicos, 17(1), 121-141. https://doi.org/10.18566/infpsic.v17n1a07

Vigotsky, L. (2016). El desarrollo del sistema nervioso. En L Quintanar-Rojas \& Y. Solovieva (Eds.), Las funciones psicológicas en el desarrollo del niño (pp. 21-36). México: Trillas.

Xomskaya, E. (2002a). El problema de los factores en la neuropsicología. Revista Española de Neuropsicología, 4(2- 
3), 151-167.

Xomskaya, E. (2002b). La escuela neuropsicológica de A.R. Luria. Revista Española de Neuropsicología, 4(2-3), 130150.

Zhinkin, N. (1960). El lenguaje. En A. Smirnov, S. Rubinstein, A. Leontiev, \& B. Tieplov (Eds.), Psicología (pp. 276-307). México: Grijalbo. 